\title{
昆明地区滇蛙两性生殖器官的生长及发育同步性
}

\author{
周 伟*，刘 钊，吴飞 \\ (西南林学院 保护生物学学院, 云南省高校森林灾害预警与控制重点实验室, 云南 昆明 650224)
}

摘要: 月平均采集并解剖滇蛙 14-16 只(雌雄约各半), 测量体长、体重、脂肪体重、精巢重、卵巢重、精巢 体积和输卵管直径等。以体长为协变量做协方差分析, 结果显示, 精巢重、卵巢重、精巢体积、输卵管直径在年 周期内差异显著, 呈周年规律性变化。滇蛙的繁殖期为 4-6 月, 5 月下旬至 6 月上旬为繁殖高峰。异速生长分析 和回归分析结果显示, 性腺发育与体重和体长多呈正异速生长 $(b>1)$, 个体增长对性腺的发育有显著影响。个体较 大的滇蛙年生殖频次至少为 2 次, 这是提高繁殖成功率的繁殖策略之一。性腺发育与脂肪体重呈负异速生长 $(b<1)$ 。 脂肪体为繁殖和冬眠提供能量储备, 脂肪体重在繁殖高峰期达最小值。少数脂肪储存不足的个体通过延迟冬眠时 间积累能量, 以便安全越冬。相关性分析和交叉相关分析结果显示, 两性生殖腺的发育具有显著的相关性, 但生 殖腺的发育并非完全同步。雄蛙精巢的发育具有一定的滞后性, 因为卵的成熟比精子的成熟需要更长的时间。但 精巢体积和输卵管直径发育具同步性, 这恰是为授精做好了准备。

关键词: 滇蛙; 生殖器官; 脂肪体; 异速生长; 同步性

中图分类号: Q959.5 文献标识码: A 文章编号: 0254-5853-(2009)01-0099-06

\section{Growth and Synchrony of Reproductive Organs in Males and Females of Rana pleuraden in Kunming Area, Yunnan}

\author{
ZHOU Wei* , LIU Zhao, WU Fei \\ (Faculty of Conservation Biology, Southwest Forestry College, Key Laboratory of Forest Disaster Warning and Control in \\ Yunnan Higher Education Institutions, Kunming Yunnan 650224, China)
}

\begin{abstract}
Fourteen to sixteen specimens of Rana pleuraden (the numbers of male and female almost equal) were collected and dissected each month. Their snout-vent length, weight of body fat, spermary and ovary, volume of spermary, and diameter of oviducts were measured. The lengths of the snout-vents were analyzed by an ANOVA as a covariate factor. The results showed that there were significant differences in the weight of spermary and ovary, volume of spermary, and diameter of oviduct, which changed annually. The breeding season of R. pleuraden appeared from April to June and the breeding peak appeared from late May to early June. The analysis of allometric and regression showed that the growth of reproductive organs was mostly positive allometric $(b>1)$ with snout-vent length or body weight. This meant that the growth of reproductive organs was substantially influenced by individual growth. The bigger individuals spawned at least twice per year, which was one of the reproductive strategies to enhance successful reproduction. The development of reproductive organs was negative allometric $(b<1)$ to the weight of body fat. Body fat provided energy for reproduction and hibernation. Weight of body fat declined to the lowest point during the breeding peak. When less fat was deposited, a few individuals would delay hibernation in order to accumulate more energy to ensure safe living through the winter. The analysis of a Pearson correlation and cross correlation showed a significant correlation in the development of reproductive organs both in males and females. However, development of reproductive organs in males and females were not completely synchronic, and the spermary grew slowly because the maturation of eggs required longer time compared to sperm. Volume of spermary developed synchronously with diameter of oviduct, which was a good preparation for insemination.
\end{abstract}

Keywords: Rana pleuraden; Reproduction organs; Fat body; Allometry; Synchrony 
繁殖季节两栖类会出现抱对、鸣叫和产卵等行 为。在外部形态上, 有的雄性婚垫变大、声囊变得 明显等。生理上则表现为性腺和输卵管变大, 生殖 细胞发育成熟, 血液中性激素含量增加等(Zhang et al, 1985; Masato, 1996; Tsai \& Tu, 2001)。生殖后蛙 的体重、性腺重、卵泡性状等均与繁殖前存在明显 差异, 繁殖器官或某些物质会随季节变化呈明显的 周年变化 (Zhou \& Sun, 1997; Li, 1998)。而随着繁 殖结束, 一切变化逐渐回归正常状态。目前关于野 生条件下蛙类生殖系统生长的年周期变化和发育 的同步性研究不多。对昭觉林蛙生殖腺及附属器官 研究表明, 该物种存在明显的年周期变化, 且雌雄 性腺的发育具同步性 (Zhou et al, 2008)。棘腹蛙的 性腺发育也具有周年变化规律, 但精巢的发育似乎 受季节变化的约束不明显 (Li, 1998)。本研究期望 通过系统观测滇蛙(Rana pleuraden)生殖器官的年 周期变化, 分析其生殖器官的生长速度及发育的同 步性, 探寻在生长过程中生殖器官的变化趋势、生 殖潜力和适应策略, 解释滇蛙在繁殖过程中的行为 适应及其生物学意义。

\section{1 材料与方法}

研究标本于 2002 年 7-11 月和 2003 年 3-6 月采自昆明市石林县西街口, 每月中或下旬采集一 次。采集的标本立即用 $5 \%$ 的甲醛溶液浸泡, 保存 待用。采集时观察并记录滇蛙栖息地生境状况。

解剖结果证明, 体长小于 $35.0 \mathrm{~mm}$ 的个体性腺 发育不成熟, 故研究标本择体长大于 $35.0 \mathrm{~mm}$ 的个 体。月均采集和解剖 14-16 只(雌雄约各半), 测量 它们的体长和称量体重(AB204-S 型电子分析天平, 精度为 $1 \mathrm{mg})$; 沿腹面剪开腹腔, 取出左侧脂肪体、 精巢或卵巢, 用电子分析天平分别称量; 将离体生 殖器官置于指形管中分别保存。

用 XTB-1 型解剖镜 (最大精度为 $0.025 \mathrm{~mm}$ ) 测 量精巢和卵巢的长径和宽径 $(\times 10$ 倍 $)$ 、输卵管直径 ( $\times 40$ 倍)和卵径 $(\times 20$ 倍)。为保证卵径测量的准确 性和减小测量误差, 随意选择解剖镜视野中相连的 15 个卵, 测量卵径大小, 取其平均值。如果卵巢中 存在颜色不同的卵, 先不分颜色测量 15 个相连卵 的卵径, 而后分别测量 15 个不同颜色卵的卵径, 最后求其平均值。

以Kolmogorov-Smironov 检验数据的正态性, 非正态数据采用 $\ln$ 转换为正态数据。用线性回归分
析、协方差分析(ANCOVA)、单因素方差分析 (ANOVA) 和最小显著差法 (Least Significant Difference, LSD)多重比较处理数据。异速生长分析 的公式为 $Y=a X^{b}$, 其中 $X$ 为自变量, $Y$ 为因变量, $a$ 为异速生长常数, $b$ 为异速生长指数。当 $b=1$ 时, $Y$ 和 $X$ 为等速生长; 当 $b>1$ 或 $b<1$ 时, $Y$ 对 $X$ 分别为正异 速生长和负异速生长(Gould, 1966)。本文分别把体 长、体重和脂肪体重定义为自变量 $X$, 其余变量为 因变量 $Y$ 。采用交叉相关分析, 分析雌雄滇蛙生殖 腺发育的同步性。所有数据处理和制图在SPSS 13.0、Excel 2003 和Eviews 5.0 中进行。

\section{2 结 果}

\section{1 生殖腺季节变化}

以体长为协变量的协方差分析结果显示, 雄蛙 精巢重量 $\left(F_{8,52}=2.477, P<0.05\right)$ 和体积 $\left(F_{8}, 52=3.219\right.$, $P<0.01)$ 、雌蛙的卵巢重量 $\left(F_{8,64}=9.864, P<0.001\right)$ 有 显著的季节性变化。单因素方差分析结果显示, 雌 蛙输卵管直径 $\left(F_{8,65}=7.650, P<0.001\right)$ 有显著的季 节性变化。最小显著差法多重比较结果表明, 雄蛙 精巢 6 月份最重, 7 月份最轻; 精巢体积亦同。雌 蛙卵巢 5 月份最重, 8 月份最轻; 输卵管直径亦同(图 1)。

\section{2 生长与异速生长}

精巢重、精巢体积、卵巢重和输卵管直径分别 与体长呈正异速生长 $(b>1)$; 卵巢重、精巢体积和输 卵管直径分别与体重呈正异速生长; 精巢重与体重 接近等速生长 $(b \approx 1)$; 䧳雄生殖腺与脂肪体重均呈 负异速生长 $(b<1)$ (表 1$)$ 。脂肪体重在 10 月最重, 6 月最轻(图 2)。

\section{3 生长与生殖腺的发育}

相关性分析结果表明, 雌蛙的输卵管直径分别 与体长和体重, 卵巢重与体重呈高度的正相关 $(r>0.70, P<0.01)$ 。雄蛙精巢重、精巢体积分别与体 长和体重呈中度的正相关 $(0.07>r>0.40, P<0.01)$ 。 雌、雄生殖腺均与脂肪体无明显的相关性 $(P<0.01)($ 表 2)。分别建立雄蛙体重与精巢重、体长 与精巢体积, 雌蛙体重与卵巢重、体长与输卵管直 径的线性回归模型, 雌雄滇蛙的生殖腺分别与各自 的体长和体重呈线性相关关系(图 3)。解剖 2003 年 6 月的 20 只雌性滇蛙, 确认 4 只大于 $58.0 \mathrm{~mm}$ 的个 体均已产过卵, 但卵巢中仍有较大的卵粒(平均卵径 $1.33 \mathrm{~mm})$ 。据卵子直径及其生长速度推测, 这 4 只 

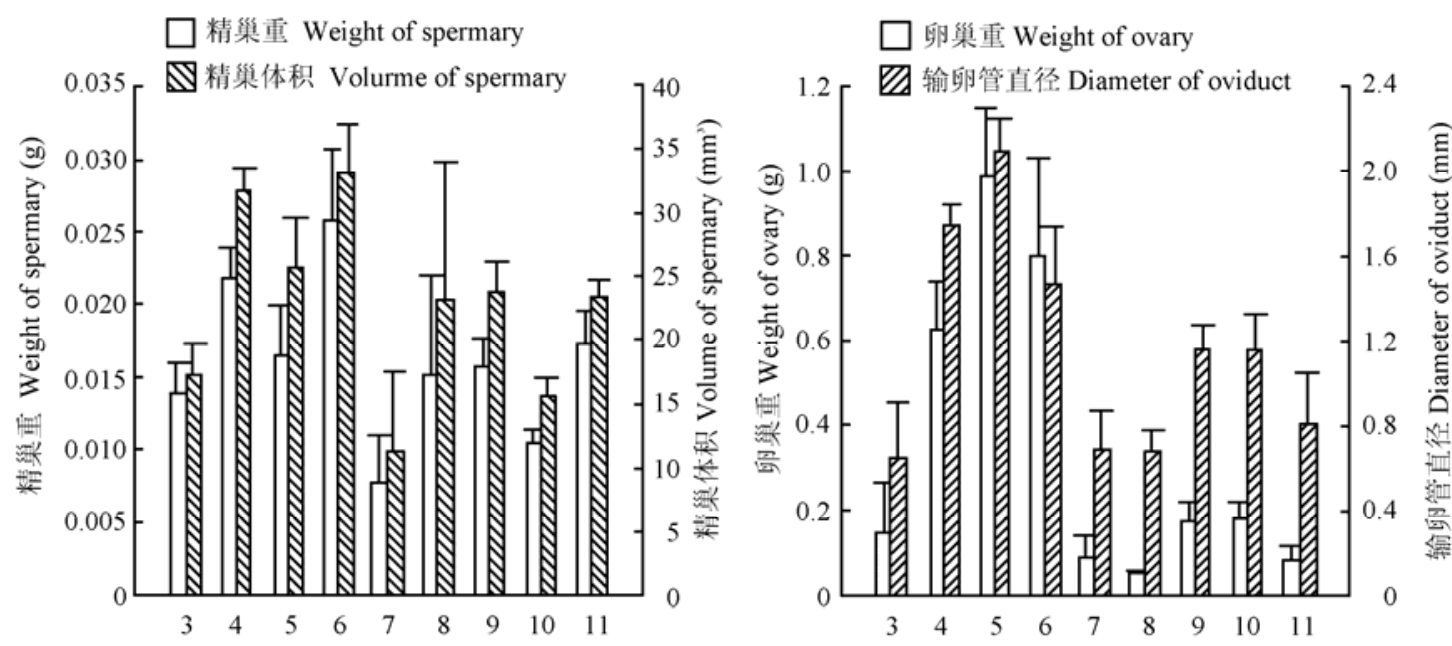

月份 Month

图 $12002-2003$ 年滇蛙生殖腺的季节变化

Fig. 1 Seasonal changes in reproductive organs of Rana pleuraden from 2002 to 2003

表 1 滇蛙生殖器官与体长、体重和脂肪体重的异速生长分析

Tab. 1 The allometric analysis between reproductive organs and body length, Weight and fat body of Rana pleuraden

\begin{tabular}{|c|c|c|c|c|c|}
\hline \multirow{2}{*}{ 自变量 $X$} & \multirow{2}{*}{ 因变量 $Y$} & \multicolumn{2}{|c|}{ 异速生长常数 $a$} & \multicolumn{2}{|c|}{ 异速生长指数 $b$} \\
\hline & & $\hat{o}$ & 우 & $\hat{o}$ & 우 \\
\hline \multirow{2}{*}{ 体长 SVL (mm) } & 精巢重 WS「卵巢重 WO (g) & $1.2 \times 10^{-7}$ & $9.3 \times 10^{-17}$ & 2.9736 & 8.7529 \\
\hline & 精巢体积 VS $\left(\mathrm{mm}^{3}\right)$ 输卵管直径 DO (mm) & $1.4 \times 10^{-5}$ & $1.9 \times 10^{-8}$ & 3.6194 & 4.4341 \\
\hline \multirow{2}{*}{ 体重 WB (mm) } & 精巢重 WSI卵巢重 WO (g) & 0.0012 & $2.7 \times 10^{-5}$ & 0.9433 & 2.9212 \\
\hline & 精巢体积 VS $\left(\mathrm{mm}^{3}\right)$ )输卵管直径 DO (mm) & 0.9842 & 0.0136 & 1.1484 & 1.4480 \\
\hline \multirow{2}{*}{ 脂肪体重 WF (g) } & 精巢重 WSI卵巢重 WO (g) & 0.0169 & 0.4876 & 0.0388 & -0.8976 \\
\hline & 精巢体积 VS $\left(\mathrm{mm}^{3}\right)$ )输卵管直径 DO (mm) & 24.1153 & 1.2416 & 0.0402 & 0.1516 \\
\hline
\end{tabular}

DO: Diameter of oviduct; SVL: Snout-vent length; VS: Volume of spermary; WB: Weight of body; WF: Weight of body fat; WO: Weight of ovary; WS: Weight of spermary.

表 2 滇蛙生长与生殖发育相关性

Tab. 2 Pearson correlation between growth and reproductive development of Rana pleuraden

\begin{tabular}{|c|c|c|c|c|}
\hline \multirow{3}{*}{ 项目 Item } & \multicolumn{4}{|c|}{ 相关系数 $r$ 值 Correlation coefficient $r$ value } \\
\hline & \multicolumn{2}{|r|}{$\hat{o}$} & \multicolumn{2}{|r|}{ 우 } \\
\hline & 精巢重(g)WS & 精巢体积 $\left(\mathrm{mm}^{3}\right) \mathrm{VS}$ & 卵巢重(g)WO & 输卵管直径 $(\mathrm{mm}) \mathrm{DO}$ \\
\hline 体长 Snout-vent length (mm) & $0.434^{* *}$ & $0.682^{* *}$ & $0.633^{* *}$ & $0.765^{* *}$ \\
\hline 体重 WB (g) & $0.442^{* *}$ & $0.676^{* *}$ & $0.716^{* *}$ & $0.802^{* *}$ \\
\hline 脂肪体重 WF (g) & -0.068 & -0.016 & -0.227 & 0.027 \\
\hline
\end{tabular}

${ }^{*} P<0.05,{ }^{* *} P<0.01$ 。表中缩写同表 1。Abbreviations in the table are the same as Tab. 1.

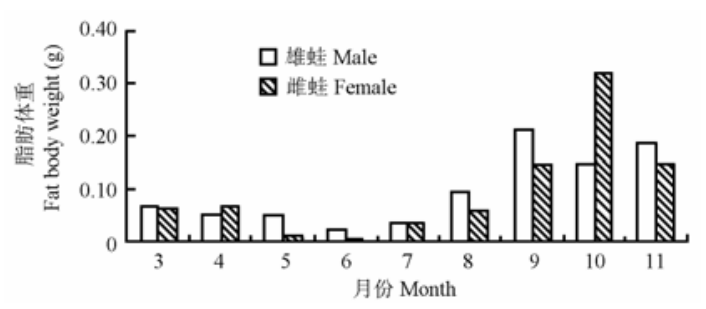

图 2 2002-2003 年滇蛙脂肪体重的季节变化

Fig. 2 Seasonal changes in fat body weight of Rana pleuraden from 2002 to 2003
滇蛙在 6 月中下旬, 最迟 7 月上旬将要再次产卵。

\section{4 生殖腺发育的同步性}

雌雄滇蛙生殖腺发育月变化的相关性分析结 果表明, 除精巢重与输卵管直径外, 精巢重与卵巢 重显著正相关 $(r=0.687, P<0.05)$, 精巢体积分别与 雌蛙卵巢重 $(r=0.776, P<0.05)$ 、输卵管直径 $(r=0.700$, $P<0.05)$ 显著正相关。精巢体积与输卵管直径的交叉 相关分析结果表明, 精巢重与卵巢重的超前滞后零 


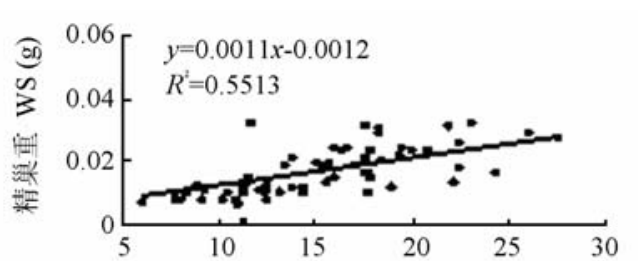

雄蛙体重 Male body weight (g)

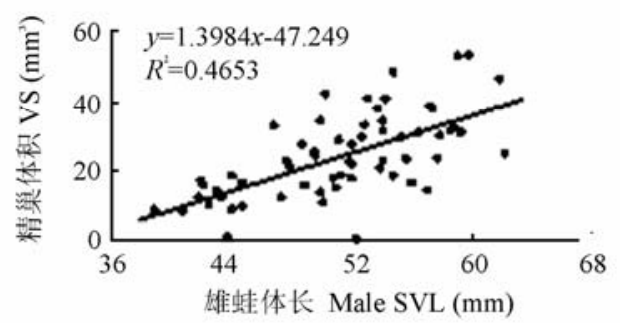

图 3 滇蛙生殖腺与体长和体重的关系

Fig. 3 Relationship between reproductive organs and snout-vent length or body weight of Rana pleuraden

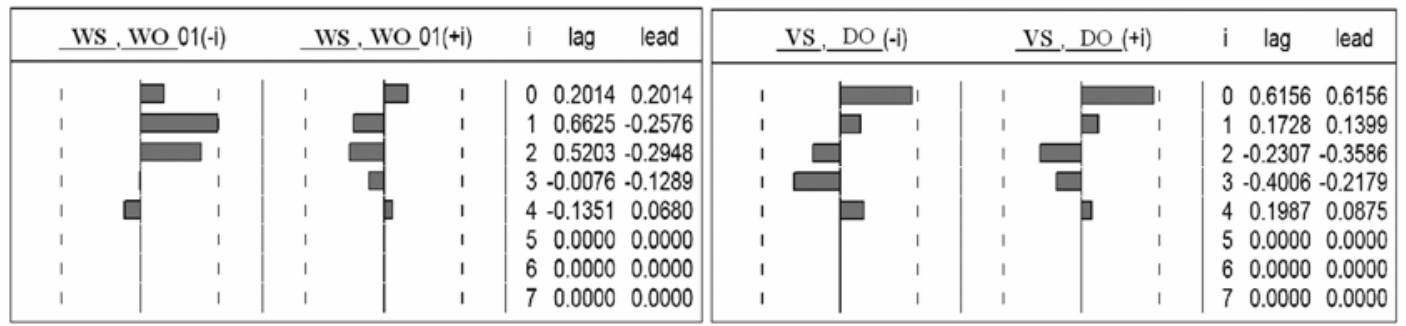

图 4 滇蛙精巢重与卵巢重(左)、精巢体积与输卵管直径(右)的交叉相关分析

Fig. 4 Cross correlation analysis between weight of spermary and ovary (left), spermary volume and oviduct diameter (right) of Rana pleuraden

每一柱状图的两侧虚线对应着正负二倍标准差。 $i$ 表示滞后期, lag 和 lead 是相应的交叉相关系数, 分别表示滞后和先行。WS, WO, VS, $\mathrm{DO}$ 含义同表 1 。

The broken symmetrical lines to histogram correspond with positive and negative double standard deviation. Letter "i" expresses the lag phase. The numbers under lag or lead are cross correlation coefficients respectively shown delay or advance. WS, WO, VS, DO are the same as in the Tab.1.

期(即原始序列, $i=0$ 时) 的相关系数不是最大值, 精巢重与卵巢重在 $1 、 2$ 期( $i=1$ 和 $i=2$ 时)的值较大, 精巢体积与输卵管直径在超前滞后零期的值较大 (图 4)。由此表明, 雌、雄滇蛙的生殖腺重量的发育 存在时滞性, 精巢体积和输卵管直径发育具同步 性。

\section{3 讨 论}

\section{1 生殖腺发育与繁殖时间}

广泛分布物种的繁殖时间随地域而异。有研究 认为, 滇蛙的繁殖期为 4-7 月 (Yang, 1991), 但 也有的认为其繁殖期为 6-7 月 (Ye et al, 1999)。 两种结论均值得商榷, 因为它们均未给出具体的地 点。滇蛙广泛分布于中国西南的云、贵、川(Ye et al,
1999)。据作者近年野外观察，在云南不同地理纬度、 不同海拔, 由于温度、湿度和降水等的差异均可导 致滇蛙的繁殖时间不同。

昆明地区滇蛙生殖与降雨有着较密切的关系。 滇蛙出蛰后，其生殖腺于 3 月份开始迅速发育，5 月份输卵管直径和卵巢重均达最大值，而 6 月份二 者均比 5 月份的小(图 1), 多无成熟或接近成熟的卵 粒, 输卵管呈淡黄色。而输卵管变黄是蛙类产卵繁 殖后的特征之一(Zhou, 1956)。野外观察和解剖学证 据均表明，滇蛙的繁殖期为 4-6 月， 5 月下旬至 6 月上旬为其繁殖高峰。滇蛙的繁殖场主要为稻田和 浅静水等区域, 昆明地区 4 月中下旬许多农田已开 始水稻育秧和耕作, 为较早繁殖的滇蛙提供了水的 条件。一般 5 月开始降雨, 恰与滇蛙的繁殖盛期吻 
合。而每年降雨的早晚、降雨量的多少则影响着多 数个体进入繁殖的时间。

\section{2 生殖腺发育与繁殖策略}

个体增长对性腺的发育有显著影响。伴随个体 生长繁殖能力逐渐提高, 一年中多次产卵是提高繁 殖成功率的繁殖策略之一。解剖结果证实, 个体较 大的滇蛙年产卵可能不止 1 次。对棘腹蛙(Rana boulengeri)、小弧斑姬蛙(Microhyla heymonsi)、大 头蛙(Rana kuhlii)等的观察证明, 它们在繁殖季节也 有多次产卵现象, 其中小弧斑姬蛙一年中产卵超过 4 次, 且第 2 次产卵量最多(Cai, 1995; Li, 1998; Tsuji \& Lue, 2000)。一年中多次产卵的滇蛙个体应是种群 中较为健壮的个体, 它们一年中多次产卵可以提高 繁殖成功率, 保证将自己的遗传信息传下去, 是成 功进化的繁殖策略之一。棘腹蛙也属于多次产卵类 型(Li, 1998)。对昭觉林蛙(Rana chaochiaoensis) 的 研究表明, 10 月为其主要的产卵时期, 11 月基本 停止产卵(Zhou et al, 2008), 且未发现其个体一年有 多次产卵。这可能与它产卵已处于冬季, 气候与食 物资源已不利于其生殖腺再次发育。

同域分布蛙类繁殖期的分化可避免产卵场和 食物资源的竞争。昆明地区的滇蛙与昭觉林蛙多呈 同域分布，但它们对生境选择和利用的差异避免了 资源竞争(Zhou et al, 2006)。更重要的是它们的繁殖 期是错开的, 滇蛙的为 4-6 月, 昭觉林蛙的为 10 月(Zhou et al, 2008)。这就更有力地保证了避免产卵 场的竞争及蝌蚪为食物资源的竞争。

\section{3 脂肪积累与繁殖和冬眠}

脂肪体为繁殖和冬眠提供能量储备。滇蛙性腺 发育与脂肪体呈负异速生长(表 1), 滇蛙脂肪体重量 的年变化与其繁殖和冬眠有极为密切的关系。对昭 觉林蛙和中华蟾蜍的研究也有类似结果(Zhou et al, 2008; Zhou \& Sun, 1997)。

滇蛙进入冬眠的时间与繁殖结束后脂肪体存 储量的多少有关。繁殖结束后, 滇蛙的脂肪体重明 显增加, 这是为安全越冬储备能量。据野外观察, 大多数滇蛙于 10 月下旬或 11 月上旬进入冬眠, 但 有少部分雌性个体 11 月下旬才冬眠。这部分个体 推迟进入冬眠时间可能与其脂肪存储量过少有关, 脂肪体过小则不足以维持整个冬眠期的能量需要,
故推迟进入冬眠时间。尽管 11 月环境条件已经严 酷, 但由于多数种类及滇蛙的大多数个体已冬眠, 此时种群密度低, 种间竞争小, 后进入冬眠的个体 获得食物的机会相对而言有所增加。然而, 11 月份 雌性滇蛙脂肪体的重量仍然比 10 月的小, 这可能 还是与当时的食物不足有关。11月下旬才进入冬眠 的雌蛙个体要比较早时候就进入冬眠的雌蛙个体 少冬眠近一个月的时间, 即后进入冬眠的雌蛙在冬 眠过程中所消耗的脂肪体重量要比早进入冬眠的 雌蛙消耗的少。因此, 相对于较早冬眠的雌蛙而言, 11 月下旬进入冬眠的雌蛙虽然存储的脂肪体较少 也能安全越冬。

\section{4 两性生殖腺发育同步性}

滇蛙两性生殖腺的发育具有显著的相关性, 但 生殖腺的发育并非完全同步。卵巢发育早，历时时 间长; 精巢的增长具有一定的滞后性(图 4)。因为与 精巢的发育相比, 卵巢中卵的成熟比精子的成熟需 要更长的时间, 所以二者重量的增加不同步。随着 卵巢的重量增加, 卵发育成熟, 输卵管膨大, 开始 为产卵做准备。与此相对应, 精巢体积快速增长, 为授精做好了准备。所以, 精巢体积和输卵管直径 发育具同步性, 这也是繁殖策略之一。

与滇蛙不同，虽然昆明地区昭觉林蛙各月间雄 性精巢重与雌性卵巢重亦呈极显著的正相关, 但两 性生殖器官的发育完全同步。其繁殖高峰期集中在 10 月, 且一年只有一次繁殖(Zhou et al, 2008)。雌 雄个体生殖器官发育的完全同步有利于节省交配 时间, 提高交配和繁殖的成功率; 也有利于卵和蝌 蚪更早的进入适宜生境, 保证成功越冬。如果发育 不同步, 面对 11 月之后更严酷的环境它将无法繁 殖。而滇蛙的繁殖高峰在每年的 5-6 月, 正值雨 季, 较好的水热和食物条件有利于雌雄蛙生殖腺充 分发育成熟。因此, 滇蛙的两性生殖腺重量发育不 同步是由于无环境胁迫的结果，发育更为成熟的卵 细胞能为后期卵裂的发生提供更多的能量储备, 有 利于受精卵的正常发育。

致谢: 西南林学院野生动植物保护利用专业 02 级硕士研究生潘晓赋协助标本采集等工作, 谨表谢 意。 


\section{参考文献:}

Cai MZ. 1995. A preliminary study on the reproductive frequency of Microhyla heymonsi Vogt [J]. Chinese Journal of Zoology, 30(5): 9-10. [蔡明章. 1995. 小弧斑姬蛙生殖频次初探. 动物学杂志, 30(5): 9-10.]

Gould SJ. 1966. Allometry and size in onto-geny and phylogeny [J]. Biol Rev, 41: 587-640.

Li WJ. 1998. The development and seasonal change of gonad Rana boulengeri [J]. Journal of Wuling, 19(3): 57-61. [李文健. 1998. 棘腹 蛙性腺的发育与周年变化. 武陵学刊, 19(3): 57-61.]

Mastao H. 1996. Seasonal fluctuations of female reproductive organs in the salamander Hynobius nigrescens [J]. Herpetologica, 52(4): 589-605.

Tsai TS, Tu MC. 2001. Reproductive cycle of female Chinese green tree vipers, Trimeresurus stejnegeri stejnegeri, in northern Taiwan [J]. Herpetologica, 57(2): 157-168.

Tsuji H, Lue KY. 2000. The reproductive ecology of female Rana (Limnonectes) kuhlii, a fanged frog of Taiwan, with particular emphasis on multiple clutches [J]. Herpetologica, 56(2): 153-165.

Yang DT. 1991. The amphibia-fauna of Yunnan [M]. Beijing: China Forestry Publishing House, 145-147. [杨大同. 1991. 云南两栖类志. 北京: 中国林业出版社, 145-147.]

Ye CY, Fei L. Hu SQ. 1999. Rare and economic amphibians of China [M]. Chengdu: Sichuan Publishing House of Science and Technology, 1-412. [叶昌媛, 费梁, 胡淑琴. 1999. 中国珍稀及经济两栖动物. 成都: 四

\section{(上接第 90 页)}

\section{3 分布及生态}

纹尾斜鳞蛇在我国的浙江、安徽、福建、台湾、 江西、河南、广西、贵州、四川以及湖南有分布。 国外尚未见有分布。在四川峨眉山、洪雅、彭县和 屏山县有分布记载。标本采集地大雪山一一位于川 滇两省四县 (四川筠连县、珙县和云南威信县、彝 良县）交汇处，属云贵高原大娄山北侧支脉，主峰 在四川省筷连县解放乡境内, 海拔约 $1780 \mathrm{~m}$, 原始 林区面积约 $2000 \mathrm{~km}^{2}$, 森林覆盖率达 $92 \%$, 属亚热 带季风型原始阔叶林区, 多雨多雾, 阴蔽潮湿。采

\section{参考文献:}

China Wildlife Conservation Association. 2002. Atlas of Reptilias of China [M] Zhenzhou: Henan Science and Technology Press. [中国野生动物 保护协会. 中国爬行动物图鉴. 郑州: 河南科学技术出版社, 198-1999.]

Guo KJ, Mi XQ, Deng XJ. 2008. Pseudoxenodon stejnegeri: A New Snake Record in FujianProvince[J]. Sichuan Journal of Zoology, 27(1): 30. [郭克疾, 米小其, 邓学建. 2008. 湖南省爬行类新记录一一花尾 斜鳞蛇. 四川动物, 27 (1): 30.]

Ji WZ. 1999. Wildling in Yunna Province[M]. Beijing: Chinese Forestry Publishing House, 108-139.[季维智. 1999. 中国云南野生动物. 北京: 中国林业出版社, 108-139.]

Tian WS, Jiang YM. 1986. China Amphibious Reptile Appraisal Handbook[M]. Beijing: Science Press, 71-133. [田婉淑, 江耀明. 1986. 中国两栖爬行动物鉴定手册. 北京: 科学出版社, 71-133.]
川科学技术出版社, 1-412.]

Zhang J, Liu JR, Cai MZ. 1985. A study on the seasonal variation in the ovary and the reproductive frequency in Rana limnocharis [J]. Acta Herpetologica Sinica, 4(4): 267-275. [张 健, 刘俊仁, 蔡明章. 1985. 泽蛙卵巢季节性变异及生殖频率的研究. 两栖爬行动物学报, 4(4): 267-275.]

Zhou BX. 1956. Anatomy of the Frog Body [M]. Beijing: Science Press, 163-168. [周本湘. 1956. 蛙体解剖. 北京: 科学出版社, 163-168.]

Zhou HM, Sun JM. 1997. The seasonal rhythm of fat content in fat-body of Chinese toad [J]. Sichuan Journal of Zoology, 16(2): 95-96. [周化民, 孙建梅. 1997. 大蟾蜍脂肪体的季节变化. 四川动物, 16(2): 95-96.]

Zhou W, Li X, Wu JP, Li FL, Li MH. 2008. Annual changes in reproductive and accompanying organs of Rana chaochiaoensis in the Kunming area [J]. Zoological Research, 29(1): 89-94. [周 伟, 李 旭, 吴建普, 李风莲, 李明会. 2008. 昆明地区昭觉林蛙生殖及附属器官的年周 期变化. 动物学研究, 29(1): 89-94.]

Zhou W, Li MH, Mai Z, Li W. 2006. Comparison of habitat utilization between sympatric population of Rana pleuraden and Rana chaochiaoensis in the Kunming area [J]. Zoological Research, 27(4): 389-395. [周 伟, 李明会, 麦 紫, 李 伟. 2006. 昆明地区滇蛙与 昭觉林蛙同域分布种群的生境利用比较. 动物学研究, 27(4): 389-395.]

集点在山间平缓的溪流边, 溪边水草茂盛, 次生的 灌木和竹林发育良好, 周围树林密被。标本发现于 废弃的公路边坡跨踏形成的土崖缝隙处。解剖发现 蛇体内有 6 枚发育的卵 (卵壳已完全形成)。

该新分布点的发现拓宽了人们对纹尾斜鳞蛇 地理分布的认识, 新分布处于云贵高原的边缘, 填 补了距离最近的两个原分布地四川南部（屏山）与 贵州（遵义的绥阳）之间的分布空白，对该物种的 动物地理学研究具有重要意义。

致谢: 宜宾学院生命科学与食品工程系 06 级 的刘凯、祝晓波等同学参加野外工作并捕获标本。

Zhao EM, Huang MH, Zhong Y. 1999. Fauna Sinica, Reptilia, Vol-III, Serpentes [M]. Beijing: Science Press, 1-460.[赵尔宓, 黄美华, 宗 愉. 1998. 中国动物志爬行纲第三卷 - 有鳞目蛇亚目. 北京: 科学出 版社, 251-253.]

Zhao EM. 2006. Snakes in China(I) [M]. Hefei: Anhui Science and Technology Publishing House, 261-261. [赵尔宓. 2006. 中国蛇类(上). 合肥: 安徽科学技术出版社, 261-261.]

Zhao EM. 2006. Snakes in China(II) [M]. Hefei: Anhui Science and Technology Publishing House, 210. [赵尔密. 2006. 中国蛇类(下). 合 肥: 安徽科学技术出版社, 210.]

Zhao EM. 2003. Coloured Atlas of Sichuan Reptiles [M]. Beijing: Chinese Forestry Publishing House, 182-183. [赵尔密. 2003. 四川爬行动物原 色图鉴. 北京: 中国林业出版社, 182-183.] 